\title{
Bone mineral density in children and young adults with neurofibromatosis type 1
}

\author{
Maya B Lodish*, Urania Dagalakis*, Ninet Sinaii ${ }^{1}$, Ethan Bornstein, \\ AeRang Kim ${ }^{2,4}$, Kelsey $B$ Lokie, Andrea M Baldwin ${ }^{2}$, James C Reynolds ${ }^{3}$, \\ Eva Dombi', Constantine A Stratakis and Brigitte C Widemann ${ }^{2}$
}

\author{
Section on Endocrinology and Genetics, Eunice Kennedy Shriver National Institute of Child Health and Human Development, \\ National Institutes of Health, Building 10-CRC, Room 1-3330, 10 Center Drive, MSC 1103, Bethesda, Maryland 20892, USA \\ ${ }^{1}$ Biostatistics and Clinical Epidemiology Service, National Institutes of Health Clinical Center, Bethesda, Maryland 20982, USA \\ ${ }^{2}$ Pharmacology and Experimental Therapeutics Section, Pediatric Oncology Branch, Center for Cancer Research, National Cancer \\ Institute, Bethesda, Maryland 20892, USA \\ ${ }^{3}$ Nuclear Medicine Division, Radiology and Imaging Sciences Department, National Institutes of Health Clinical Center, Bethesda, \\ Maryland 20892, USA \\ ${ }^{4}$ Department of Oncology, Children's National Medical Center, Washington, District of Columbia 20010, USA \\ (Correspondence should be addressed to M B Lodish; Email: lodishma @mail.nih.gov) \\ *(M B Lodish and U Dagalakis contributed equally to this work)
}

\begin{abstract}
Concern for impaired bone health in children with neurofibromatosis type 1 (NF-1) has led to increased interest in bone densitometry in this population. Our study assessed bone mineral apparent density (BMAD) and whole-body bone mineral content (BMC)/height in pediatric patients with NF-1 with a high plexiform neurofibroma burden. Sixty-nine patients with NF-1 (age range 5.2-24.8; mean $13.7 \pm 4.8$ years) were studied. Hologic dual-energy $X$-ray absorptiometry scans (Hologic, Inc., Bedford, MA, USA) were performed on all patients. BMD was normalized to derive a reference volume by correcting for height through the use of the BMAD, as well as the BMC. BMAD of the lumbar spine (LS 2-4), femoral neck (FN), and total body BMC/height were measured and Z-scores were calculated. Impaired bone mineral density was defined as a Z-score $\leq-2$. Forty-seven percent of patients exhibited impaired bone mineral density at any bone site, with $36 \%$ at the LS, $18 \%$ at the FN, and $20 \%$ total BMC/height. BMAD Z-scores of the LS $(-1.60 \pm 1.26)$ were more impaired compared with both the FN $(-0.54 \pm 1.58 ; P=0.0003)$ and the whole-body BMC/height $Z$-scores $(-1.16 \pm 0.90 ; P=0.036)$. Plexiform neurofibroma burden was negatively correlated with LS BMAD $\left(r_{\mathrm{s}}=-0.36, P=0.01\right)$. In pediatric and young adult patients with NF-1, LS BMAD was more severely affected than the FN BMAD or whole-body $\mathrm{BMC} /$ height.
\end{abstract}

Endocrine-Related Cancer (2012) 19 817-825

\section{Introduction}

The bone health of children and adolescents has become an increasingly recognized medical concern, and a number of genetic disorders of childhood are associated with impaired bone density. Neurofibromatosis type 1 (NF-1) is a variably penetrant autosomal dominant disorder with a birth incidence of $\sim 1$ in 2700 and prevalence of 1 in 4560 (Evans et al. 2010). NF-1 is caused by mutations in the neurofibromin gene on chromosome 17q11.2 (Marchuk et al. 1991). NF-1 is characterized by dermal and plexiform neurofibromas (PN) as well as café au lait macules, axillary and inguinal freckling, optic gliomas, and Lisch nodules (Gutmann et al. 1997, Lynch \& Gutmann 2002, Ward \& Gutmann 2005). Other common features within the diagnostic criteria are osseous abnormalities such as sphenoid wing dysplasia and tibial pseudarthrosis. Additional skeletal defects include bone cysts, spinal canal widening, vertebral body narrowing, rib-penciling, vertebral scalloping, and dural ectasia (Stevenson et al. 2007). Impaired BMD has been reported in several studies of children with NF-1; however, not all studies corrected for short stature (Dulai et al. 2007, Stevenson et al. 2007, 
Yilmaz et al. 2007, Brunetti-Pierri et al. 2008, Duman et al. 2008, Caffarelli et al. 2010).

In children and adolescents, the diagnosis of osteoporosis is made by a combination of densitometric criteria plus the presence of both a clinically significant fracture history (Lewiecki et al. 2008). Impaired BMD in children is defined as a BMD Z-score that is $\leq-2.0$, adjusted for age, gender, and body size. Reduced peak bone mass has been linked to an increased risk of osteoporosis earlier in adult life (Hui et al. 1990). NF-1 has been associated with compromised bone health in childhood that may leave patients vulnerable to fracture. Yilmaz et al. (2007) found that 31 pediatric NF-1 patients (mean age $9.9 \pm 4.1$ years) with skeletal involvement were more likely to have a lumbar spine (LS) areal BMD (aBMD) lower than -2 compared with a control group. Using dual-energy X-ray absorptiometry (DXA) in 84 pediatric NF-1 patients (mean age $10.3 \pm 4.1$ years), Stevenson et al. (2007) found that with or without skeletal abnormality NF-1 patients had a significant decrease in aBMD compared with control subjects. Dulai et al. (2007) studied 23 NF-1 patients (mean age 10.8 years) and showed that $30 \%$ had age-matched $Z$-scores below -1.5 for total body BMD. Duman et al. (2008) using DXA in 32 patients (mean age $8.38 \pm 3.75$ years) showed significantly decreased BMD of the LS and femoral neck (FN) areas in NF-1 patients compared with controls. Similarly, BrunettiPierri et al. (2008) found significantly lower LS BMD in $73 \mathrm{NF}-1$ patients (mean age 16.0 years) compared with controls while Caffarelli et al. (2010) found significantly decreased $\mathrm{FN}$ aBMD and femur aBMD in 55 NF-1 patients (mean age $9.3 \pm 5.4$ years) vs controls; both studies also corrected for height.

The impaired bone density in NF-1 may be associated with an increased risk of fracture. Fractures are a common occurrence in childhood, with an incidence rate in the general population ranging from 1.2 to $5 \%$ depending on the study (Jones 2004, Rennie et al. 2007, Donaldson et al. 2008). One study has evaluated the fracture rate in children with NF-1. Fracture risk was evaluated in 103 Finnish children ages 3-16 years with NF-1 and were compared with age- and gender-matched controls. Children with NF-1 had a fracture incidence of 12/1000 person years vs an incidence of seven in control children, equal to a relative risk ratio of 3.4 (95\% CI 1.5-7.8; Heerva et al. 2012).

One factor that may contribute to impaired BMD is 25-hydroxyvitamin D deficiency. Lammert et al. (2006) showed that 55 NF-1 patients had a mean serum 25-hydroxyvitamin D concentration of $14.0 \mathrm{ng} / \mathrm{ml}$ compared with $31.4 \mathrm{ng} / \mathrm{ml}$ in 58 healthy controls. Seitz et al. (2010) studied a subgroup of four adult NF-1 patients, who underwent DXA scans a year after oral administration of 25 -hydroxyvitamin D supplements and showed significant improvement in spinal BMD Z-scores.

Chronic disease, poor nutrition, and tumor burden associated with NF-1 are additional factors that may affect BMD. In addition, calculations of BMD are usually underestimated in short individuals, particularly children, which necessitates the use of the bone mineral apparent density (BMAD) and whole-body bone mineral content (BMC) to correct for height. Many studies documenting a high prevalence of impaired BMD in pediatric NF-1 patients have failed to account for short stature in growing children, which leads to erroneous interpretation. The primary objective of this project was to characterize BMAD in pediatric NF-1 patients accounting for gender, age, ethnicity, and stature. Secondary aims sought to examine the prevalence of 25-hydroxyvitamin D deficiency in the patient cohort. Additionally, we analyzed the contribution of the PN tumor burden, scoliosis, scalloping, dural ectasia, and Eastern Cooperative Oncology Group Performance (ECOG) status to the BMAD. Our study is unique in that it is the first to examine the potential relationship between PN tumor burden and bone mineral density in children with NF-1.

\section{Materials and methods}

\section{Subjects of protocol}

Data were collected from a cohort of 69 children and young adults (ages 5-24 years) with a confirmed clinical diagnosis of NF-1 enrolled in a longitudinal study via retrospective chart review (National Institutes of Health Consensus Development Conference 1988). Collected baseline characteristics were gender, age, ethnicity, stature, ECOG performance score, 25-hydroxyvitamin D levels, and presence or absence of skeletal abnormalities (scoliosis, scalloping, dural ectasia, and history of fracture). All study tests were performed as part of the NF-1 Natural History study for children and adolescents (NCT00924196). Consent was obtained from each patient over 18 years, while parental consent and patient assent was obtained for those individuals under age 18 years after full explanation of the purpose and nature of all procedures used. The investigation was approved the Institutional Review Board of the Pediatric Oncology Branch, National Cancer Institute, at the NIH. 


\section{Assessment of bone density}

Hologic DXA scans were analyzed to measure bone density. LS BMD of patients with spinal hardware was excluded because the devices cause artifacts on the DXA scan. Impaired BMAD for chronological age was defined as having a $Z$-score $\leq-2.00$ (Gordon et al. 2008). Owing to the fact that BMD calculations are often underestimated in shorter pediatric patients, the BMD was normalized by correcting the values to adjust for differences in bone size through the use of the BMAD measurement, an estimation of volumetric bone density $\left(\mathrm{g} / \mathrm{cm}^{3}\right)$. Values for BMC and area were used to calculate BMAD. BMAD $Z$-scores were calculated using published normative data for Hologic, as available on the Stanford applet: http://www-statclass.stanford.edu/pediatric-bones (Bachrach et al. 1999). The normative data for the Stanford applet ages ranged from 9 to 25 years and is adjusted for age, gender, and race.

\section{Hormone assays}

25-Hydroxyvitamin D levels were defined as severely deficient $(<10 \mathrm{ng} / \mathrm{ml})$, mild-to-moderately deficient (10-24 ng/ml), and normal (25-80 ng/ml). Laboratory data assessing parathyroid hormone (PTH), calcium, 25-hydroxyvitamin $\mathrm{D}$, and alkaline phosphatase were conducted within 6 months of the DXA. Elevated spot urinary calcium/creatinine ratio was defined as $\geq 0.3$.

\section{Tumor measurements}

All patients had their PN tumor burden measured using the heuristics algorithm developed at NIH to analyze patient data through the MEDx (v3.42) visualization and analysis software (Sensor Systems, Inc., Sterling, VA, USA) using region-based and boundary-based segmentation (Solomon et al. 2004). Tumor burden was measured in cubic centimetre and percent tumor burden was calculated by dividing the tumor burden over the patient's body weight in kilogram.

\section{Performance status}

ECOG performance status is validated using scales and criteria are used to assess how a patient's disease affects their daily living abilities (Oken et al. 1982). In our cohort, scores ranged from 0 (fully active, able to carry on all pre-disease performance without restriction), 1 (restricted in physically strenuous activity but ambulatory and able to carry out work of a light or sedentary nature), to 2 (ambulatory and capable of all self-care but unable to carry out any work activities). ECOG status was assessed by interviews with study participants by a single nurse practitioner.

\section{Statistical analysis}

Baseline patient characteristics were analyzed as descriptive variables and summary statistics were generated. Paired $t$-tests were used to compare paired data (e.g. BMAD Z-scores at different sites), and two sample $t$-tests were used for comparing two independent groups. Tests for correlation included Pearson's correlation coefficient or Spearman's rho, as appropriate. The relationship between tumor burden and LS BMAD was explored with logistic and linear regression modeling. For logistic modeling, BMAD $Z$-scores were categorized as normal $(>-2)$ vs impaired $(\leq-2)$. The potential effects of patient characteristics hypothesized to be related to BMD such as age, gender, body mass index (BMI), Z-score, Tanner stage, prior PN therapy, and ECOG were considered in these models. Results are presented as mean \pm s.D., unless otherwise indicated. As appropriate, multiple comparisons were adjusted by the stepdown Bonferroni's method. A two-sided $P \leq 0.05$ was considered statistically significant. Data were analyzed using SAS System Software version 9.2 (SAS Institute, Inc., Cary, NC, USA).

\section{Results}

Baseline characteristics are described in Table 1. One distinguishing characteristic of our patient cohort is the high prevalence PN, because these tumors have been known to develop in about $50 \%$ of individuals with NF-1; therefore, the $94 \%$ rate of PN in our study cohort is atypical (Tonsgard et al. 1998, Thakkar et al. 1999, Tucker et al. 2009). Seventy-one percent of the total patient cohort had been or were currently enrolled in one or multiple clinical trials with investigational drugs used to treat their PN. The most widely used therapies in our patient cohort included tipifarnib, peginterferon $\alpha-2 b$, pirfenidone, and sirolimus, with $41,39,26$, and $13 \%$ of patients having received therapy with one of these agents respectively.

Patients had a low height SDS $(-0.92 \pm 1.17)$. While the majority of patient's laboratory data remained within the normal range (Table 1), it is notable that eight patients had elevated urine calcium levels, two patients had elevated PTH levels, and three patients had elevated alkaline phosphatase levels. Within the patient cohort, 30 (47.6\%) had normal 25-hydroxyvitamin D status and $33(52.4 \%)$ had 
M B Lodish, U Dagalakis et al.: BMAD in pediatric NF1 patients

Table 1 Patient characteristics. All data are mean \pm s.D., unless otherwise indicated

\begin{tabular}{|c|c|c|c|c|c|}
\hline Females (\%) & $29(42 \%)$ & & & & \\
\hline Males (\%) & $40(58 \%)$ & & & & \\
\hline Age (years) & $13.7 \pm 4.8$ & & & & \\
\hline \multicolumn{6}{|l|}{ Race (\%) } \\
\hline Asian & $1(1 \%)$ & & & & \\
\hline African-American & $3(4 \%)$ & & & & \\
\hline White & $58(84 \%)$ & & & & \\
\hline White/African & $1(1 \%)$ & & & & \\
\hline Hispanic & $4(6 \%)$ & & & & \\
\hline Other & $2(3 \%)$ & & & & \\
\hline Height SDS & $-0.92 \pm 1.17$ & & & & \\
\hline BMI Z-score & $0.03 \pm 1.2$ & & & & \\
\hline Tanner stages & & & & Male & Female \\
\hline I & & & & $18(45 \%)$ & $9(31 \%)$ \\
\hline II & & & & $5(13 \%)$ & $2(7 \%)$ \\
\hline III & & & & $0(0 \%)$ & $1(3 \%)$ \\
\hline IV & & & & $1(2 \%)$ & $1(3 \%)$ \\
\hline V & & & & $15(38 \%)$ & $16(55 \%)$ \\
\hline No data & & & & $1(2 \%)$ & \\
\hline $\begin{array}{l}\text { Tumor burden in cubic centimetre (median, } \\
\text { 25th-75th percentiles) }\end{array}$ & \multicolumn{3}{|l|}{$781(259-1734)$} & & \\
\hline $\begin{array}{l}\text { Percent tumor burden (cc/kg; median, } \\
\text { 25th-75th percentiles) }\end{array}$ & \multicolumn{3}{|l|}{$2(0.53-4.23)$} & & \\
\hline Prior fracture history & \multicolumn{3}{|l|}{$14(20 \%)$} & & \\
\hline Scoliosis present & \multicolumn{3}{|l|}{$42(61 \%)$} & & \\
\hline Scalloping present & \multicolumn{3}{|l|}{$32(46 \%)$} & & \\
\hline Dural ectasia present & \multicolumn{3}{|l|}{$38(55 \%)$} & & \\
\hline Moderate 25-hydroxyvitamin D deficiency ${ }^{a}$ & \multicolumn{3}{|l|}{$33(52 \%)$} & & \\
\hline PTH (nl: $16-87$ pg/ml) & \multicolumn{3}{|l|}{$39.4 \pm 20.9$} & & \\
\hline Serum calcium (nl: $2.05-2.50 \mathrm{mmol} / \mathrm{l})$ & \multicolumn{3}{|l|}{$2.3 \pm 0.1$} & & \\
\hline Urine calcium/creatinine ratio & \multicolumn{3}{|l|}{$0.2 \pm 0.1$} & & \\
\hline 25 Vitamin D2 and D3 (nl: 25-80 ng/ml) & \multicolumn{3}{|l|}{$25.4 \pm 8.5$} & & \\
\hline \multicolumn{6}{|l|}{ Alkaline phosphatase (U/l) } \\
\hline Age (years) & Male & Female & Age (years) & Male & Female \\
\hline $4-6$ & 93-309 & 96-297 & $4-6$ & $170 \pm 70$ & $155 \pm 40$ \\
\hline $7-9$ & $86-315$ & $69-325$ & $7-9$ & $161 \pm 59$ & $142 \pm 39$ \\
\hline $10-12$ & $42-362$ & $51-332$ & $10-12$ & $207 \pm 48$ & $171 \pm 25$ \\
\hline $13-15$ & $74-390$ & $50-162$ & $13-15$ & $153 \pm 58$ & $117 \pm 84$ \\
\hline $16-18$ & $52-171$ & $47-119$ & $16-18$ & $106 \pm 48$ & $80 \pm 22$ \\
\hline$>18$ & $37-116$ & $37-116$ & $>18$ & $75 \pm 18$ & $61 \pm 14$ \\
\hline
\end{tabular}

$\mathrm{nl}$, standard normal values used for these measurements.

${ }^{a}$ Moderate 25-hydroxyvitamin D deficiency is defined as $10-24 \mathrm{ng} / \mathrm{ml}$.

moderate 25-hydroxyvitamin D deficiency. There were 14 patients $(20.3 \%)$ with prior fractures and 42 patients $(60.9 \%)$ had scoliosis.

Of the 69 patients, final data were available for FN BMAD in 53 patients, for LS BMAD in 50 patients, and for BMC/height in 51 patients; LS BMAD was excluded in three patients because of previously installed hardware, 12 because of age (i.e. under 9 years and applet did not allow for $Z$-score calculation), and one because of both, while three patients did not have a BMD measured at this site. Without correcting for short stature and using BMD values of $\leq-2$ at any site to define impaired bone density, the prevalence of impaired BMD in this group would have been overestimated at $56 \%$. In this study, $47 \%$ of patients had impaired BMAD at any bone site, while the expected frequency in the normal population is $2.3 \%$ at each individual bone site based on the normal distribution. The breakdown of impaired BMAD in our cohort consists of $36 \%$ at the LS, $18 \%$ at the FN, and $20 \%$ total $\mathrm{BMC} /$ height. BMAD Z-scores of the LS $(-1.60 \pm 1.26)$ were lower than those of the $\mathrm{FN}$ $(-0.54 \pm 1.58 ; P=0.0003$; Fig. 1$)$. The $\mathrm{BMC} /$ height $Z$-scores $(-1.16 \pm 0.90)$ were worse than those of the FN $(-0.54 \pm 1.58 ; P=0.007)$, while only slightly better than those of the LS $(-1.60 \pm 1.26 ; P=0.036)$ (Fig. 1). Tumor burden as a percent of body weight was inversely correlated with both the LS BMAD Z-scores 


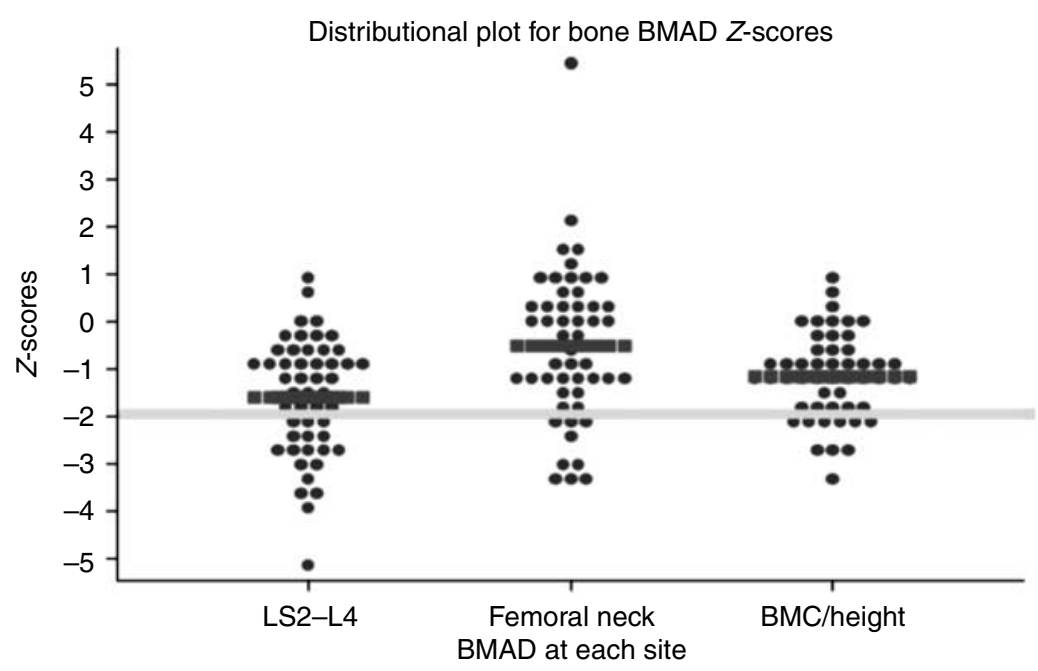

Figure 1 Distributional plot for lumbar spine (LS2-L4) and femoral neck BMAD and BMC/height Z-scores. Thick yellow line is the -2.00 level $Z$-score. Thick red bars represent mean $Z$-scores.

$\left(r_{\mathrm{s}}=-0.36, P=0.01\right)$ and $\mathrm{BMC} /$ height $\left(r_{\mathrm{s}}=-0.28\right.$, $P=0.048$ ) (Fig. 2). The odds ratio of having a greater tumor burden was $1.26(95 \%$ CI 1.006-1.575, $P=0.04)$ in patients with LS BMAD $Z$-scores $\leq-2$. Increased tumor burden, age, BMI Z-score, and Tanner stage were statistically significantly associated with impaired LS BMAD by univariable regression analysis, while gender, prior PN therapy, and ECOG score were not (Table 2). When the relation between tumor burden and LS BMAD was adjusted for these covariates, only BMI $Z$-score remained statistically significantly $(P=0.002)$ associated with impaired BMAD. We asked the obvious question of whether patients with NF-1 and impaired BMAD were more likely to have a fracture than those NF-1 patients with normal BMAD. The likelihood of having a prior fracture was not statistically different in patients classified as having any BMAD Z site $Z$-score $\leq-2$ vs those with normal BMAD.

As secondary analyses, we investigated the contribution of additional skeletal abnormalities and 25-hydroxyvitamin D deficiency to BMAD in NF-1 patients. Individuals with scoliosis had lower BMAD $Z$-scores of the FN than the patients without scoliosis $(P=0.007$; Table 3$)$. Patients with scalloping or dural ectasia had worse LS BMAD Z-scores when compared with individuals without these defects $(-1.61 \pm 1.17$ vs $-0.31 \pm 0.11, P<0.0001)$. Interestingly, the presence of a PN involving the LS was not associated with worse LS BMAD Z-scores in our study. Even though $52 \%$ of patients had moderate 25-hydroxyvitamin $\mathrm{D}$ deficiency, there were no correlations with BMAD Z-scores for any of the three skeletal sites.

\section{Discussion}

Previous studies have analyzed bone density in patients with NF-1 (Dulai et al. 2007, Stevenson et al. 2007, Yilmaz et al. 2007, Brunetti-Pierri et al. 2008, Duman et al. 2008, Caffarelli et al. 2010). While more data exist for adults, a number of smaller studies have looked at impaired BMAD in children with NF-1, but not all of them corrected for height. Our study is unique in its examination of a large pediatric and young adult population with a significant PN burden, while looking at BMAD Z-scores to correct for discrepancies in height.

Despite all that is known about neurofibromin, it is still unclear how skeletal abnormalities evolve in these patients. Investigations in a mouse model of NF-1 by Yu et al. (2005) suggested that there was increased apoptosis of osteoprogenitors in the affected mice. Another study has shown increased osteoclast activity in NF-1 mice correlated with increased p21 function (Yang et al. 2006). A study of bone metabolism markers found that 26 children with NF-1 had lower levels of osteocalcin compared with normal controls (Duman et al. 2008). The Seitz et al. (2010) study looked at iliac crest biopsies in $14 \mathrm{NF}-1$ adult patients and found decreased trabecular thickness, as well as increased osteoblast and osteoclast activity, demonstrating increased bone turnover when compared with controls.

We found a $47 \%$ prevalence of impaired BMD among pediatric NF-1 patients. The results show that the LS was more affected with a $36 \%$ prevalence of impaired BMD and a mean BMAD Z-score of -1.7 . The LS is predominantly composed of trabecular bone, 


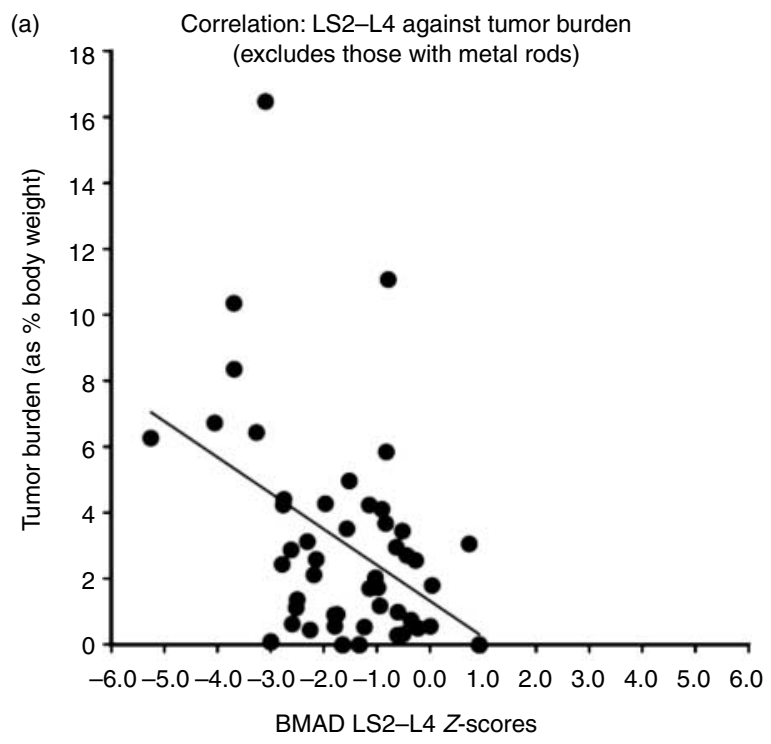

(b) Correlation: BMC/height against tumor burden

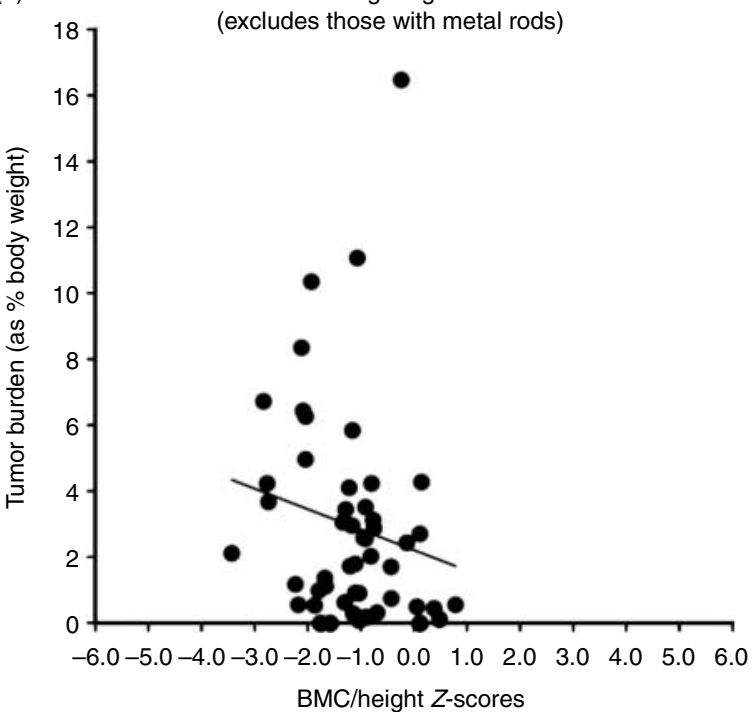

Figure 2 (a) Correlation plot of tumor burden as a percent of body weight inversely correlated with the lumbar spine BMAD Z-scores $\left(r_{\mathrm{s}}=-0.36, P=0.01\right)$. Negative correlation marked by thin black line. (b) Correlation plot of tumor burden as a percent of body weight inversely correlated with the BMC/height $\left(r_{\mathrm{s}}=-0.28\right.$, $P=0.048)$. Negative correlation marked by thin black line.

compared with the FN that is primarily cortical bone. The pattern of more pronounced bone density impairment in the LS has been seen previously in other studies in pediatric patients with NF-1. BrunettiPierri et al. showed that $48 \%$ of patients had impaired LS BMD Z $Z$-scores using the criteria of a $Z$-score $\leq-1.5$ (Brunetti-Pierri et al. 2008). Yilmaz et al. (2007) found that $12.5 \%$ of the pediatric patients had low LS BMD Z-scores based on a Z-scores criteria of $\leq-2$. One potential explanation for low LS BMD is that trabecular bone of the LS may be more sensitive to mineralization problems in growing children (Brunetti-Pierri et al. 2008). These patients may potentially be at risk for asymptomatic vertebral compression fractures given the low LS BMD; however, this has yet to be investigated. Our study also showed that certain skeletal abnormalities including scoliosis, vertebral scalloping, and dural ectasia were associated with lower BMAD Z-scores. Scoliosis affects up to $26 \%$ of individuals with NF-1, which could magnify the effects of impaired BMD (Friedman 2002). Interestingly, our NF-1 patients had a relatively low reported frequency of fracture $(20.3 \%)$ compared with the percentage of fractures in the normal pediatric population in which the $42 \%$ of boys and $27 \%$ of girls report sustaining at least one fracture by the age of 16 years (Brinker \& O’Connor 2004).

Our results indicated a correlation of larger tumor burden with a lower BMAD Z-score; however, the correlation was not strong. In addition to increased tumor burden, age, BMI Z-score, and Tanner stage were also statistically significantly associated with impaired LS BMAD by univariable analysis. A potential explanation for the correlation between tumor burden and decreased BMAD is that the PN may secrete a substance that has a negative impact on bone health, but this has not been investigated. Other mesenchymal tumors secrete fibroblast growth factor 23 as a paraneoplastic phenomenon, which may be part of the explanation of decreased bone density in patients with larger PN tumor burden (Ovesen et al. 2003, Ginde et al. 2009). However, arguing against this hypothesis is the normal serum calcium and PTH levels in these patients. Another hypothesis to explain the association of impaired BMAD with PN is linked to the pathogenesis of PN development in NF-1. As neurofibromin is a tumor suppressor, a second-hit mutation precedes the development of tumors including PN; mutations that predispose these individuals to develop PN may potentially affect bone if they occur early in development (Staser et al. 2012). When other

Table 2 Univariable regression modeling of potential contributing factors in the relationship between tumor burden and LS BMAD

\begin{tabular}{lll}
\hline Variable & $\boldsymbol{R}^{\mathbf{2}}$ & $\boldsymbol{P}$ value \\
\hline Gender & 0.05 & 0.15 \\
ECOG & 0.05 & 0.13 \\
Age & 0.23 & $0.0009^{*}$ \\
Tanner stage & 0.30 & $0.0001^{*}$ \\
BMl Z-score & 0.30 & $0.0001^{*}$ \\
Prior therapy & 0.01 & 0.44 \\
\hline
\end{tabular}

*Statistically significant. 
Table 3 BMAD Z-scores of patients with and without scoliosis

\begin{tabular}{lrrl}
\hline Site & $\begin{array}{c}\text { Patients with- } \\
\text { out scoliosis } \\
\text { (mean } \pm \text { s.D.) }\end{array}$ & $\begin{array}{c}\text { Patients with } \\
\text { scoliosis } \\
\text { (mean } \pm \text { s.D.) }\end{array}$ & $\boldsymbol{P}$ value \\
\hline Lumbar spine & $-1.55 \pm 1.10$ & $-1.64 \pm 1.38$ & 0.8 \\
Femoral neck & $0.17 \pm 1.68$ & $-1.01 \pm 1.34$ & 0.0066 \\
BMC/height & $-1.15 \pm 0.79$ & $-1.17 \pm 0.99$ & 0.9 \\
\hline
\end{tabular}

factors were considered together, only BMI Z-score was statistically significantly associated with impaired $\mathrm{BMAD}$, providing evidence that a number of variables work together to contribute to bone density. This could also indicate the effects of decreased physical activity as a contributor to poor bone health.

Despite the $52 \%$ of patients with moderate 25-hydroxyvitamin $\mathrm{D}$ deficiency, which we initially hypothesized would be associated with low bone density, no correlation was found between these two measures in the patient cohort. It is unclear how other factors might have affected 25-hydroxyvitamin D levels, as latitude, season, and skin tone have been shown to influence 25-hydroxyvitamin D levels (Ovesen et al. 2003, Ginde et al. 2009). Further investigation and longitudinal studies are warranted to better understand the relationship between 25-hydroxyvitamin $\mathrm{D}$ and bone density in pediatric NF-1 patients.

One limitation of our study is the potential effects of tumor therapies on BMD. Within our patient cohort, $91 \%$ had PN, and the majority of these individuals were enrolled on clinical trials with investigational drugs for their PN. Studies have shown that several of these cancer treatments can negatively impact bone health, while a number of them are new agents without known bone-related effects. Methotrexate has been found to inhibit bone formation by blocking bone turnover and inhibiting the early differentiation of osteoblasts, which can potentially lead to methotrexate osteopathy characterized by osteoporosis, fractures, and bone pain (Holzer et al. 2003, Wilson et al. 2012). However, only two out of the 69 patients in our cohort received methotrexate treatment. Another limitation of our study is that a higher prevalence of PN in our cohort may make our results less generalizable to patients with NF-1 as a whole.

Understanding and identifying the elevated risks of low BMD in patients with NF-1 are critical in order to provide improved patient care. There are several ways that patients can minimize their risk such as participating in weight-bearing exercise, optimizing their calcium and 25-hydroxyvitamin D intake, and avoiding osteotoxic medications. The use of bisphosphonates in pediatric patients to improve BMD is controversial due to potential longitudinal affects on bone health and lack of randomized controlled studies (Marini 2003). Comprehensive management of the pediatric patient with NF-1 includes assessment of skeletal health, and our study provides important additional information about bone mineral density in this patient population.

\section{Declaration of interest}

The authors declare that there is no conflict of interest that could be perceived as prejudicing the impartiality of the research reported.

\section{Funding}

This work was supported by the Intramural programs of the National Cancer Institute and the Eunice Kennedy Shriver National Institute of Child Health and Human Development of the National Institutes of Health.

\section{References}

National Institutes of Health Consensus Development Conference 1988 National Institutes of Health Consensus Development Conference Statement: neurofibromatosis. Bethesda, Maryland, USA, July 13-15, 1987. Neurofibromatosis 1 172-178.

Bachrach LK, Hastie T, Wang MC, Narasimhan B \& Marcus R 1999 Bone mineral acquisition in healthy Asian, Hispanic, black, and Caucasian youth: a longitudinal study. Journal of Clinical Endocrinology and Metabolism 84 4702-4712. (doi:10.1210/jc.84.12.4702)

Brinker MR \& O'Connor DP 2004 The incidence of fractures and dislocations referred for orthopaedic services in a capitated population. Journal of Bone and Joint Surgery. American Volume 86 290-297.

Brunetti-Pierri N, Doty SB, Hicks J, Phan K, Mendoza-Londono R, Blazo M, Tran A, Carter S, Lewis RA, Plon SE et al. 2008 Generalized metabolic bone disease in neurofibromatosis type I. Molecular Genetics and Metabolism 94 105-111. (doi:10.1016/j. ymgme.2007.12.004)

Caffarelli C, Gonnelli S, Tanzilli L, Vivarelli R, Tamburello S, Balestri P \& Nuti R 2010 Quantitative ultrasound and dual-energy X-ray absorptiometry in children and adolescents with neurofibromatosis of type 1 . Journal of Clinical Densitometry 13 77-83. (doi:10.1016/ j.jocd.2009.10.002)

Donaldson LJ, Reckless IP, Scholes S, Mindell JS \& Shelton NJ 2008 The epidemiology of fractures in England. Journal of Epidemiology and Community Health 62 174-180. (doi:10.1136/jech.2006.056622)

Dulai S, Briody J, Schindeler A, North KN, Cowell CT \& Little DG 2007 Decreased bone mineral density in 
neurofibromatosis type 1: results from a pediatric cohort. Journal of Pediatric Orthopedics 27 472-475. (doi:10.1097/01.bpb.0000271310.87997.ae)

Duman O, Ozdem S, Turkkahraman D, Olgac ND, Gungor F \& Haspolat S 2008 Bone metabolism markers and bone mineral density in children with neurofibromatosis type-1. Brain \& Development 30 584-588. (doi:10.1016/j. braindev.2008.02.002)

Evans DG, Howard E, Giblin C, Clancy T, Spencer H, Huson SM \& Lalloo F 2010 Birth incidence and prevalence of tumor-prone syndromes: estimates from a UK family genetic register service. American Journal of Medical Genetics. Part A 152A 327-332. (doi:10.1002/ ajmg.a.33139)

Friedman JM 2002 Neurofibromatosis 1: clinical manifestations and diagnostic criteria. Journal of Child Neurology 17 548-554 (discussion 571-542, 646-551). (doi:10.1177/088307380201700802)

Ginde AA, Liu MC \& Camargo CA Jr 2009 Demographic differences and trends of vitamin D insufficiency in the US population, 1988-2004. Archives of Internal Medicine 169 626-632. (doi:10.1001/archinternmed.2008.604)

Gordon CM, Bachrach LK, Carpenter TO, Crabtree N, El-Hajj Fuleihan G, Kutilek S, Lorenc RS, Tosi LL, Ward KA, Ward LM et al. 2008 Dual energy X-ray absorptiometry interpretation and reporting in children and adolescents: the 2007 ISCD Pediatric Official Positions. Journal of Clinical Densitometry 11 43-58. (doi:10.1016/j.jocd.2007.12.005)

Gutmann DH, Aylsworth A, Carey JC, Korf B, Marks J, Pyeritz RE, Rubenstein A \& Viskochil D 1997 The diagnostic evaluation and multidisciplinary management of neurofibromatosis 1 and neurofibromatosis 2 .

Journal of the American Medical Association 278 51-57. (doi:10.1001/jama.1997.03550010065042)

Heerva E, Koffert A, Jokinen E, Kuorilehto T, Peltonen S, Aro HT \& Peltonen J 2012 A controlled register based study of 460 neurofibromatosis 1 (NF1) patients: increased fracture risk in children and adults over 41 years. Journal of Bone and Mineral Research 27 2333-2337.

Holzer G, Krepler P, Koschat MA, Grampp S, Dominkus M \& Kotz R 2003 Bone mineral density in long-term survivors of highly malignant osteosarcoma. Journal of Bone and Joint Surgery. British Volume 85 231-237. (doi:10.1302/0301-620X.85B2.13257)

Hui SL, Slemenda CW \& Johnston CC Jr 1990 The contribution of bone loss to postmenopausal osteoporosis. Osteoporosis International 1 30-34. (doi:10.1007/ BF01880413)

Jones G 2004 Growth, children, and fractures. Current Osteoporosis Reports 2 75-78. (doi:10.1007/s11914-0040014-2)

Lammert M, Friedman JM, Roth HJ, Friedrich RE, Kluwe L, Atkins D, Schooler T \& Mautner VF 2006 Vitamin D deficiency associated with number of neurofibromas in neurofibromatosis 1. Journal of Medical Genetics 43 810-813. (doi:10.1136/jmg.2006.041095)
Lewiecki EM, Gordon CM, Baim S, Leonard MB, Bishop NJ, Bianchi ML, Kalkwarf HJ, Langman CB, Plotkin H, Rauch F et al. 2008 International Society for Clinical Densitometry 2007 adult and pediatric official positions. Bone 43 1115-1121. (doi:10.1016/j.bone.2008.08.106)

Lynch TM \& Gutmann DH 2002 Neurofibromatosis 1. Neurologic Clinics 20 841-865. (doi:10.1016/S07338619(01)00019-6)

Marchuk DA, Saulino AM, Tavakkol R, Swaroop M, Wallace MR, Andersen LB, Mitchell AL, Gutmann DH, Boguski M \& Collins FS 1991 cDNA cloning of the type 1 neurofibromatosis gene: complete sequence of the NF1 gene product. Genomics 11 931-940. (doi:10.1016/08887543(91)90017-9)

Marini JC 2003 Do bisphosphonates make children's bones better or brittle? New England Journal of Medicine 349 423-426. (doi:10.1056/NEJMp038103)

Oken MM, Creech RH, Tormey DC, Horton J, Davis TE, McFadden ET \& Carbone PP 1982 Toxicity and response criteria of the Eastern Cooperative Oncology Group. American Journal of Clinical Oncology 5 649-655. (doi:10.1097/00000421-198212000-00014)

Ovesen L, Andersen R \& Jakobsen J 2003 Geographical differences in vitamin D status, with particular reference to European countries. Proceedings of the Nutrition Society 62 813-821. (doi:10.1079/ PNS2003297)

Rennie L, Court-Brown CM, Mok JY \& Beattie TF 2007 The epidemiology of fractures in children. Injury $\mathbf{3 8}$ 913-922. (doi:10.1016/j.injury.2007.01.036)

Seitz S, Schnabel C, Busse B, Schmidt HU, Beil FT, Friedrich RE, Schinke T, Mautner VF \& Amling M 2010 High bone turnover and accumulation of osteoid in patients with neurofibromatosis 1. Osteoporosis International 21 119-127. (doi:10.1007/s00198-0090933-y)

Solomon J, Warren K, Dombi E, Patronas N \& Widemann B 2004 Automated detection and volume measurement of plexiform neurofibromas in neurofibromatosis 1 using magnetic resonance imaging. Computerized Medical Imaging and Graphics 28 257-265. (doi:10.1016/j. compmedimag.2004.03.002)

Staser K, Yang FC \& Clapp DW 2012 Pathogenesis of plexiform neurofibroma: tumor-stromal/hematopoietic interactions in tumor progression. Annual Review of Pathology 7 469-495. (doi:10.1146/annurev-pathol011811-132441)

Stevenson DA, Moyer-Mileur LJ, Murray M, Slater H, Sheng X, Carey JC, Dube B \& Viskochil DH 2007 Bone mineral density in children and adolescents with neurofibromatosis type 1. Journal of Pediatrics 150 83-88. (doi:10.1016/j.jpeds.2006.10.048)

Thakkar SD, Feigen U \& Mautner VF 1999 Spinal tumours in neurofibromatosis type 1: an MRI study of frequency, multiplicity and variety. Neuroradiology 41 625-629. (doi:10.1007/s002340050814) 
Tonsgard JH, Kwak SM, Short MP \& Dachman AH 1998 CT imaging in adults with neurofibromatosis-1: frequent asymptomatic plexiform lesions. Neurology $\mathbf{5 0}$ 1755-1760. (doi:10.1212/WNL.50.6.1755)

Tucker T, Friedman JM, Friedrich RE, Wenzel R, Funsterer C \& Mautner VF 2009 Longitudinal study of neurofibromatosis 1 associated plexiform neurofibromas. Journal of Medical Genetics 46 81-85. (doi:10.1136/jmg.2008. 061051)

Ward BA \& Gutmann DH 2005 Neurofibromatosis 1: from lab bench to clinic. Pediatric Neurology 32 221-228. (doi:10.1016/j.pediatrneurol.2004.11.002)

Wilson CL, Dilley K, Ness KK, Leisenring WL, Sklar CA, Kaste SC, Stovall M, Green DM, Armstrong GT, Robison LL et al. 2012 Fractures among long-term survivors of childhood cancer: a report from the Childhood Cancer Survivor Study. Cancer [in press].

Yang FC, Chen S, Robling AG, Yu X, Nebesio TD, Yan J, Morgan T, Li X, Yuan J, Hock J et al. 2006

Hyperactivation of $\mathrm{p} 21$ ras and PI3K cooperate to alter murine and human neurofibromatosis type 1-haploinsufficient osteoclast functions. Journal of Clinical Investigation 116 2880-2891. (doi:10.1172/ JCI29092)

Yilmaz K, Ozmen M, Bora Goksan S \& Eskiyurt N 2007 Bone mineral density in children with neurofibromatosis 1. Acta Paediatrica 96 1220-1222. (doi:10.1111/j.16512227.2007.00401.x)

Yu X, Chen S, Potter OL, Murthy SM, Li J, Pulcini JM, Ohashi N, Winata T, Everett ET, Ingram D et al. 2005 Neurofibromin and its inactivation of Ras are prerequisites for osteoblast functioning. Bone 36 793-802. (doi:10.1016/j.bone.2005.01.022)

Received in final form 15 October 2012

Accepted 19 October 2012

Made available online as an Accepted Preprint 22 October 2012 